\title{
OPERATOR-STABLE PROBABILITY DISTRIBUTIONS ON VECTOR GROUPS
}

\author{
BY \\ MICHAEL SHARPE( $\left.{ }^{1}\right)$
}

1. Introduction. An operator-stable probability distribution in a group $G$ is a limit law arising, roughly speaking, from affine modification of the partial sums of a sequence of independent identically distributed $G$-valued random variables. This paper is concerned with a more palpable description of an operator-stable distribution in the case where $G$ is a vector group; i.e. the topological group underlying a $d$-dimensional real vector space.

The first step is to reduce the problem to that of finding those probability measures $\lambda$, all of whose convolution powers are of the same type; i.e. for each integer $k \geqq 1$, there is an automorphism $B_{k}$ of $G$ such that $\lambda^{k}$ is a translation of the measure $\lambda \circ B_{k}^{-1}$. It is then shown that $\lambda$ is operator-stable if and only if for each $t>0, \lambda^{t}$ is a translation of the measure $\lambda \circ[\exp (\log t \cdot B)]^{-1}$, for an automorphism $B$ of $G$ characterized by conditions on the spectrum of $B$ regarded as a linear operator on a vector space.

2. Notation and definitions. Throughout this paper, we denote by $V$ a $d$-dimensional vector group. In several proofs, we shall use the same symbol to denote the same group with the additional structure of a vector space, or even an inner product space. Denote by $\mathscr{P}=\mathscr{P}(V)$ the set of probability measures on $V$. With the topology of weak convergence, and multiplication defined by convolution, $\mathscr{P}$ becomes a topological semigroup. We denote convolution of two measures $\lambda, \mu$ by $\lambda * \mu$, and the $n$th convolution power of $\lambda$ by $\lambda^{n}$.

By $V^{\wedge}$, we mean the character group of $V$, "identified" with the dual vector space of the vector space $V$. In the sequel, $x$ will denote the generic element of $V$ and $y$ the generic element of $V^{\wedge}$. Let $(x, y)$ denote the bilinear pairing of $V$ and $V^{\wedge}$ brought about by $y$ acting as a linear functional on $x$. As a group character, $y$ acts on $x$ according to $\exp i(x, y)$. The characteristic function of a measure $\lambda \in \mathscr{P}(V)$ is defined by

$$
\varphi(y)=\lambda^{\wedge}(y)=\int_{V} \exp i(x, y) \lambda(d y) .
$$

Given $\lambda \in \mathscr{P}(V)$, we define $\lambda^{-} \in \mathscr{P}(V)$ by $\lambda^{-}(E)=\lambda(-E)$. The mapping $\lambda \rightarrow \lambda^{-}$ is an involutive automorphism of $\mathscr{P}$. It is easy to check that $\lambda^{-\wedge} \equiv \lambda^{\wedge}-$, the last

Received by the editors October 12, 1967.

(1) Research partially supported by NSF-GP-3509 at Yale University. This paper is drawn from the author's dissertation presented for the degree of Doctor of Philosophy in Yale University. 
bar denoting the complex conjugate. The measure $\lambda$ is called symmetric if and only if $\lambda=\lambda^{-}$. For any $\lambda \in \mathscr{P}$, the measure ${ }^{\circ} \lambda=\lambda * \lambda^{-}$is symmetric, and is called the symmetrization of $\lambda$.

Let $H(\lambda)=\left\{y \in V^{\wedge} \mid \lambda^{\wedge}(y)=1\right\}$. It is easy to see that $H(\lambda)$ is a closed subgroup of $V^{\wedge}$. Note that $\left\{y \in V^{\wedge}|| \lambda^{\wedge}(y) \mid=1\right\}=H\left({ }^{c} \lambda\right)$ is a closed subgroup of $V^{\wedge}$ containing $H(\lambda)$.

Denote by $S(\lambda)$ the support of the measure $\lambda \in \mathscr{P}(V)$, viz., the smallest closed set $F$ in $V$ with $\lambda(F)=1$. The relation $S(\lambda * \mu)=\mathrm{Cl}(S(\lambda)+S(\mu))$ is well known. We call a measure $\lambda \in \mathscr{P}$ full if and only if $S(\lambda)$ is not contained in any $(d-1)$-dimensional hyperplane of $V$. If $\lambda$ is not full, it is called deficient.

Proposition 1. $\lambda \in \mathscr{P}(V)$ is a full measure if and only if $H\left({ }^{\circ} \lambda\right)$ does not contain a 1-dimensional subgroup of $V^{\wedge}$.

Proof. Firstly, $\lambda$ is full if and only if ${ }^{\circ} \lambda$ is full. If $S\left({ }^{\circ} \lambda\right) \subset X$, a $(d-1)$-dimensional subspace of $V$, then

$$
\begin{aligned}
{ }^{\circ} \lambda^{\wedge}(y) & =\int_{X} \exp i(x, y)^{\circ} \lambda(d x) \\
& =1 \text { if } y \in X^{\perp}=\left\{y \in V^{\wedge} \mid(x, y)=0, \forall x \in X\right\},
\end{aligned}
$$

and $X^{\perp}$ is a 1-dimensional subspace of $V^{\wedge}$. Conversely, if $H\left({ }^{\circ} \lambda\right)$ contains a 1dimensional subspace $Y$ of $V^{\wedge}$, then $\int_{V} \exp i(x, y)^{\circ} \lambda(d x)=1$ for all $y \in Y$ implies $\exp i(x, y)=1,{ }^{\circ} \lambda$-almost everywhere, for all $y \in Y$, which implies

so that

$$
{ }^{\circ} \lambda\{x \mid \exp i(x, y)=1\}=1 \text { for all } y \in Y
$$

$S\left({ }^{\circ} \lambda\right) \subset\{x \mid(x, y)=0 \bmod 2 \pi$ for all $y \in Y\}=\{x \mid(x, y)=0$ for all $y \in Y\}=Y^{\perp}$, a $(d-1)$-dimensional subspace of $V$.

We mention that the set $\mathscr{F}$ of full measures in $\mathscr{P}(V)$ is an open subsemigroup of $\mathscr{P}$.

Let us denote by End $V$ the ring of continuous endomorphisms of the group $V$, identified with the ring of linear transformations of the vector space $V$. Let Aut $V$ denote the group of continuous automorphisms of $V$, identified with the group $\mathrm{Gl}(V)$ of nonsingular linear transformations of the vector space $V$. For any $A \in$ End $V$ and $\lambda \in \mathscr{P}(V)$, let $A \lambda$ denote the measure $A \lambda(F)=\lambda\left(A^{-1}(F)\right), F$ a Borel subset of $V$. If $A \in$ Aut $V$ and $a \in V$, we call the mapping $\lambda \rightarrow A \lambda * \delta(a)$ an affine transformation, $\delta(a)$ denoting the point mass at $a$. If instead $A \in$ End $V \sim$ Aut $V$, the mapping will be called singular affine. The set of affine transformations $(A, a)$ is denoted by Aff $V$. If $x$ is a $V$-valued random variable having distribution $\mu$, then clearly, $A x+a$ has distribution $A \lambda * \delta(a)$.

It is not difficult to verify that for any bounded continuous function $f$ on $V$,

$$
\int_{V} f d(A \lambda)=\int_{V} f \circ A d \lambda
$$


and that $S(A \lambda)=A S(\lambda),(A B) \lambda=A(B \lambda), A(\lambda * \mu)=A \lambda * A \mu$ and $(A \lambda)^{\wedge}(y)=\lambda^{\wedge}\left(A^{*} y\right)$, where $A^{*}$ denotes the adjoint map of $V^{\wedge} \rightarrow V^{\wedge}$ induced by the bilinear pairing $(\cdot, \cdot)$; i.e. $(A x, y) \equiv\left(x, A^{*} y\right)$. We give End $V$ the compact-open topology as a set of functions from $V$ to $V$. This topology is equivalent to the norm topology of End $V$ as a space of linear operators on $V$, when $V$ is provided with a vector norm. It is easy to check that the mapping $\langle A, \lambda\rangle \rightarrow A \lambda$ of End $V \times \mathscr{P}(V) \rightarrow \mathscr{P}(V)$ is jointly continuous. We define an equivalence relation in $\mathscr{P}(V)$ by writing $\lambda \sim \mu$ if and only if there is an affine transformation $(A, a)$ such that $\lambda=A \mu * \delta(a)$. The measures $\lambda, \mu$ are then said to be of the same type, and an equivalence class of measures is called a type. Clearly, random variables $x$ and $A x+a$ will have distributions of the same type.

3. Statement of the problem. In terms of random variables, the problem we study is enunciated as follows: suppose that $\left\{x_{n}\right\}$ is a sequence of $V$-valued random variables with common distribution $\mu$, and assume that the terms of the sequence are mutually independent; assume, further, that $\left(A_{n}, a_{n}\right)$ is a sequence of affine transformations of $V$ such that the distribution of $A_{n}\left(x_{1}+\cdots+x_{n}\right)+a_{n}$ converges to a measure $\lambda \in \mathscr{P}(V)$; what can be said about the limit measure $\lambda$ ?

Converting this to a problem involving only measures, we ask which measures $\lambda$ can arise as limits of sequences $A_{n} \mu^{n} * \delta\left(a_{n}\right)$.

Our aim is to characterize those $\lambda$ which are full and are such limits-denote by $\mathscr{S}$ the class of such measures. For reasons which do not become clear until the problem is posed in a different form, $\mathscr{S}$ is called the class of operator-stable measures on $V$. It is clear that $\mathscr{S}$ is invariant under affine transformations.

We refer the reader to Feller [1] for an account of the class $\mathscr{S}$ when $V$ reduces to the real line. It is possible in this case to give an explicit formula for the characteristic function of a stable distribution. In fact (Feller [1, p. 542]) any such characteristic function $\lambda^{\wedge}$ must have the form $\lambda^{\wedge}(y)=\exp \left(a \psi_{\alpha, \delta}(y)+i b y\right)$, where $0<\alpha \leqq 2$, $-1 \leqq \delta \leqq 1$, and

$$
\begin{aligned}
\psi_{\alpha, \delta}(y) & =-|y|^{\alpha}[1-i \operatorname{sgn} y \delta \tan \pi \alpha / 2], & & \alpha \neq 1, \\
& =-|y|[1+i \operatorname{sgn} y \delta \log |y|], & & \alpha=1 .
\end{aligned}
$$

In particular, $\lambda^{\wedge} t(y)=\lambda^{\wedge}\left(t^{1 / \alpha} y\right) \exp i b(t) y, t>0$ where $b(t)$ is real. It is easily seen that if $\alpha \neq 1$, there exists $b$ such that $\mu=\lambda * \delta(b)$ satisfies $\mu^{\wedge t}(y)=\mu^{\wedge}\left(t^{1 / \alpha} y\right)$ but if $\alpha=1$, it is not generally possible to make such a centering, and $b(t)$ has the form $b(t)=c t \log t$. These results are obtained from the fact that the Khintchine-Lévy measure $M$ (see next paragraph) of a stable distribution must be given by $M\{[x, \infty)\}$ $=c p x^{-\alpha}, M\{(-\infty,-x]\}=c q x^{-\alpha}$ where $c \geqq 0, p+q=1, p \geqq 0$ and $q \geqq 0$.

All that has been done so far in the multi-dimensional case is to find limits of distributions of sequences $A_{n}\left(x_{1}+\cdots+x_{n}\right)+a_{n}$ where $A_{n}$ is a multiple of the identity operator. By the same techniques as in the one-dimensional case, one finds the Khintchine-Lévy representing measures as mixtures of one-dimensional $\mathrm{K}-\mathrm{L}$ measures for stable distributions concentrated in rays starting at the origin. 
Our results will provide analogues of these facts, except that an explicit representation for a general operator-stable characteristic function does not seem possible.

4. The Khintchine-Lévy formula. A stable distribution is infinitely divisible and a major tool in the analysis of $\mathscr{S}$ will be the multi-dimensional form of the Khintchine-Lévy representation. The form we use is a slight modification of the original Lévy [4] version, and we state it as

Proposition 2 (KhintChine-LÉvy). To an infinitely divisible measure $\lambda \in \mathscr{P}(V)$, there corresponds a triple $(c, \phi, M)$ consisting of an element $c \in V$, a nonnegative quadratic form $\phi$ on $V$ and a nonnegative Radon measure $M$ on the locally compact space $V \sim\{0\}$ satisfying

(i) $M$ is finite off every neighborhood of 0 , and

(ii) $\int_{K \sim\{0\}}\|x\|^{2} M(d x)<\infty$ for every compact subset $K$ of $V,\|\cdot\|$ being any vector norm on $V$, such that

$$
\lambda^{\wedge}(y)=\exp \left\{i(c, y)-\phi(y)+\int_{V \sim\{0\}}[\exp i(x, y)-1-i(\tau(x), y)] M(d x)\right\} .
$$

Here, $\tau: V \rightarrow V$ is any continuous function satisfying

(a) $\tau(x)=x+O\left(\|x\|^{2}\right)(x \rightarrow 0)$, and

(b) $\tau$ is bounded.

$A$ change in the function $\tau$ produces only a change in the term $c$.

For uniqueness, we know that if, for $j=1,2$,

$$
\psi_{j}(y)=i\left(c_{j}, y\right)-\phi_{j}(y)+\int_{V \sim\{0\}}\left[\exp i(x, y)-1-i\left(\tau_{j}(x), y\right)\right] M_{j}(d x)
$$

and $\psi_{1}(y)=\psi_{2}(y)$ for all $y \in V^{\wedge}$, then $\phi_{1}=\phi_{2}$ and $M_{1}=M_{2}$. If, further, $\tau_{1}=\tau_{2}$, then $c_{1}=c_{2}$.

We describe this representation by saying that $\lambda$ has representing triple $(c, \phi, M)$. Any nonnegative Radon measure $M$ on $V \sim\{0\}$ satisfying (i) and (ii) will be called a K-L measure. Note that the element $c$ determines merely a translation of $\lambda$, and $\phi$ determines the Gaussian component.

The next proposition is pure calculation, and sets out the manner in which the representing triple changes with an affine transformation of $\lambda$.

Proposition 3. If $\lambda$ is infinitely divisible in $\mathscr{P}(V)$ and has representing triple $(c, \phi, M)$, and if $A \in$ Aut $V$ and $a \in V$, then the representing triple of $A \lambda * \delta(a)$ is $\left(c^{\prime}, \phi \circ A^{*}, A M\right)$ for some $c^{\prime} \in V$.

Proof. In the calculation, it is only necessary to note that if $\tau$ satisfies (a) and (b) of Proposition 2, then so does the function $A \circ \tau \circ A^{-1}$.

Finally, if $\lambda$ is infinitely divisible with representing triple $(c, \phi, M)$ and $t>0$, we let $\lambda^{t}$ denote the $t$ th power of $\lambda$; viz., the infinitely divisible measure with representing triple $(t c, t \phi, t M)$. The semigroup $\left\{\lambda^{t} \mid t>0\right\}$ is then weakly continuous. 
5. Reduction of the problem. The following lemma is used repeatedly in the sequel. It is a generalization of a lemma which is well known in the one-dimensional set-up (see e.g. Feller [1, p. 246, Lemma 1]).

Proposition 4 (The Compactness Lemma). Suppose that for $n=1,2, \ldots$, $\lambda_{n} \in \mathscr{P}(V),\left(A_{n}, a_{n}\right)$ is an affine transformation, and assume that

(i) $\lambda_{n} \rightarrow \lambda \in \mathscr{P}(V)$,

(ii) $A_{n} \lambda_{n} * \delta\left(a_{n}\right) \rightarrow \mu \in \mathscr{P}(V)$.

Then, if $\lambda$ and $\mu$ are full in $V$, the set $\left\{A_{n} \mid n=1,2, \ldots\right\}$ is precompact in Aut $V$, $\left\{a_{n} \mid n=1,2, \ldots\right\}$ is precompact in $V$, and if $A$ and a are limit points in these respective sets, then $A \lambda * \delta(a)=\mu$.

In other words, if for $n=1,2, \ldots, \lambda_{n} \sim \mu_{n}$, if $\lambda_{n} \rightarrow \lambda, \mu_{n} \rightarrow \mu$ and $\lambda$ and $\mu$ are full, then $\lambda \sim \mu$.

Proof. Let $\langle\cdot, \cdot\rangle$ be an inner product on $V$, and let $\|x\|^{2}=\langle x, x\rangle$. If it is possible to prove that

(A) $\left\{a_{n} \mid n=1,2, \ldots\right\}$ is bounded in $V$, and

(B) $\left\{\left\|A_{n}\right\| \mid n=1,2, \ldots\right\}$ is bounded in $R$,

then any sequence $\left\{n_{k}\right\}$ of positive integers will have a subsequence $\left\{n_{k}^{\prime}\right\}$ such that $a_{n_{k}^{\prime}}$ converges to an element $a$ of $V$, and $A_{n_{k}^{\prime}}$ converges to an element $E$ of End $V$. By joint continuity,

$$
A_{n_{k}} \lambda_{n_{k}} * \delta\left(a_{n \dot{k}}\right) \rightarrow E \lambda * \delta(a) \text { as } k \rightarrow \infty \text {. }
$$

But the left side converges to $\mu$, so $\mu=E \lambda * \delta(a)$. Since $S(\mu)=E S(\lambda)+a$ is not contained in a $(d-1)$-dimensional hyperplane, $E$ must be invertible, so that all limit points of $\left\{A_{n} \mid n=1,2, \ldots\right\}$ are in Aut $V$, showing $\left\{A_{n} \mid n=1,2, \ldots\right\}$ to be precompact in Aut $V$. It suffices, therefore, to prove (A) and (B).

We start with (B). Firstly, the conditions (i) and (ii) imply that ${ }^{\circ} \lambda_{n} \rightarrow{ }^{\circ} \lambda$ and $A_{n}\left({ }^{\circ} \lambda_{n}\right) \rightarrow{ }^{\circ} \mu$, where ${ }^{\circ} \lambda$ and ${ }^{\circ} \mu$ are full. In proving (B), we may therefore assume that $a_{n}=0$ for all $n$. With this assumption in force temporarily, we shall assume, for purposes of obtaining a contradiction, that $\left\{\left\|A_{n}\right\| \mid n=1,2, \ldots\right\}$ is not bounded.

Let us choose a fixed orthonormal basis for the inner product space $(V,\langle\cdot, \cdot\rangle)$, and think of the operators $A_{n}$ as matrices, relative to this basis. Now, $A_{n}$ can be factored into polar form, $A_{n}=U_{n} P_{n}$, where $P_{n}$ is positive self-adjoint and $U_{n}$ is orthogonal. The fact that $P_{n}$ is diagonalizable by orthogonal matrices implies that $A_{n}=V_{n} D_{n} W_{n}$ where $D_{n}$ is diagonal, and $V_{n}$ and $W_{n}$ are orthogonal. Passing to a subsequence, if necessary, it may be assumed that one entry of $D_{n}$ tends to infinity as $n \rightarrow \infty$. Modifying $V_{n}$ if necessary, it may be assumed that it is the first entry, $\alpha_{n}^{-1}$, which does so. Then $\alpha_{n} \rightarrow 0$. Since the orthogonal group is compact, it may be assumed that $W_{n}$ converges to an orthogonal matrix $W$. Set $\nu_{n}=W_{n} \lambda_{n}$. We have $A_{n} W_{n}^{-1}\left(\nu_{n}\right)=A_{n} \lambda_{n} \rightarrow \mu$ and $\nu_{n} \rightarrow W \lambda=\nu$, also full. Replacing $\lambda_{n}$ and $A_{n}$ by $\nu_{n}$ and $A_{n} W_{n}^{-1}$, we see that it may be assumed that $A_{n}$ can be factored into the form 
$V_{n} D_{n}, V_{n}$ orthogonal and $D_{n}$ diagonal. Let $\varepsilon>0$ be given: let $\Delta_{r}=\{x \mid\|x\| \leqq r\}$ and let $r$ be chosen so large that

(iii) $\lambda_{n}(\Delta)>1-\varepsilon / 2$ for all $n$,

(iv) $A_{n} \lambda_{n}(\Delta)>1-\varepsilon / 2$ for all $n$,

(v) $\Delta$ is a continuity set for $\lambda$ and $\mu$ (i.e. $\lambda($ bdry $\Delta)=\mu($ bdry $\Delta)=0$ ).

Now, $A_{n}^{-1}(\Delta)=D_{n}^{-1} V_{n}^{-1}(\Delta)=D_{n}^{-1}(\Delta)$, so by (iii) and (iv),

$$
\lambda_{n}\left(D_{n}^{-1}(\Delta) \cap \Delta\right)>1-\varepsilon \text { for all } n,
$$

and

$$
\lambda_{n}\left(D_{n}^{-1}(\Delta)\right) \rightarrow \mu(\Delta)>1-\varepsilon .
$$

Notice that $x \in D_{n}^{-1}(\Delta) \cap \Delta$ implies $\|x\| \leqq r$ and $\left\|D_{n} x\right\| \leqq r$ so that $\left|x_{j}\right| \leqq r$ for $j=2, \ldots, d$ and $\left|x_{1}\right| \leqq r \alpha_{n}$. Let $L_{n}$ be the rectangle

$$
\left\{x|| x_{1}\left|\leqq \alpha_{n} r,\right| x_{j} \mid \leqq r \text { for } j=2, \ldots, d\right\} .
$$

Then $D_{n}^{-1}(\Delta) \cap \Delta \subset L_{n}$. Define $f_{K}(x)=\max \left[\left(1-K\left|x_{1}\right|, 0\right)\right], K>0$. We have

$$
\begin{aligned}
\int_{V} f_{K}(x) \lambda_{n}(d x) & \geqq \int_{L_{n}} f_{K}(x) \lambda_{n}(d x) \\
& \geqq \min \left\{f_{K}(x) \mid x \in L_{n}\right\} \cdot \lambda_{n}\left(L_{n}\right) \\
& \geqq \lambda_{n}\left(D_{n}^{-1}(\Delta) \cap \Delta\right) \cdot\left(1-K \alpha_{n} r\right) \\
& >(1-\varepsilon)\left(1-K \alpha_{n} r\right) \text { for all } n, K>0 .
\end{aligned}
$$

Letting $n \rightarrow \infty$, we find that $\int_{V} f_{K}(x) \lambda(d x) \geqq 1-\varepsilon$ for all $K>0$, hence

$$
\lambda\left\{x|| x_{1} \mid \geqq 1 / K\right\} \geqq 1-\varepsilon \text { for all } K>0,
$$

implying $\lambda\left\{x \mid x_{1}=0\right\} \geqq 1-\varepsilon$. Since $\varepsilon>0$ is arbitrary, $\lambda\left\{x \mid x_{1}=0\right\}=1$ and $\lambda$ must be deficient. This contradiction implies the truth of (B).

The proof of (A) now proceeds as follows: by assumption, $\left\{A_{n} \lambda_{n} * \delta\left(a_{n}\right)\right\}$ is precompact, and since (B) is true, $\left\{A_{n} \lambda_{n}\right\}$ is precompact; there is therefore a compact subset $C$ of $V$ such that $A_{n} \lambda_{n}(C)>3 / 4$ and $\left(A_{n} \lambda_{n} * \delta\left(a_{n}\right)\right)(C)>3 / 4$ for all $n$, so that $A_{n} \lambda_{n}\left(C \cap\left(C-a_{n}\right)\right)>1 / 2$ and certainly $C \cap\left(C-a_{n}\right) \neq \varnothing$; we conclude that for all $n,\left|a_{n}\right| \leqq \operatorname{diam} C<\infty$, and (A) is proven.

Corollary 1. If $\lambda$ is any full measure in $\mathscr{P}(V)$ and if we define Inv $\lambda=\{(A, a) \in$ Aff $V \mid A \lambda * \delta(a)=\lambda\}$, then Inv $\lambda$ is a compact subgroup of Aff $V$.

COROLlaRy 2. In the open subsemigroup $\mathscr{F}$ of full measures in $\mathscr{P}(V)$, the relation " " induces compact equivalence classes.

Both of these corollaries follow trivially from the compactness lemma.

6. A characterization of operator-stable measures. The class $\mathscr{S}$ will now be characterized in a manner more amenable to analysis than that of the original definition. 
THEOREM 1. A full measure $\mu \in \mathscr{P}(V)$ is stable (i.e., $\mu \in \mathscr{S}$ ) if and only if for each integer $n \geqq 1$, there is an affine transformation $\left(B_{n}, b_{n}\right)$ such that $\mu^{n}=B_{n} \mu * \delta\left(b_{n}\right)$. In other words, $\mu$ is in $\mathscr{S}$ if and only if $\mu$ is full and all powers of $\mu$ are of the same type.

Proof. Firstly, if all powers of $\mu$ are of the same type, then for each $n$,

$$
\mu=C_{n} \mu^{n} * \delta\left(c_{n}\right), \quad\left(C_{n}, c_{n}\right) \in \text { Aff } V
$$

so $\mu=\lim C_{n} \mu^{n} * \delta\left(c_{n}\right)$ implying $\mu \in S$.

Suppose now that $\mu$ is full and that

(i) $\mu=\lim A_{n} \lambda^{n} * \delta\left(a_{n}\right), \lambda \in \mathscr{P}(V),\left(A_{n}, a_{n}\right) \in$ Aff $V$.

Since convolution is a jointly continuous operation, we obtain from (i) the equation

$$
\mu^{m}=\lim _{n}\left[A_{n} \lambda^{n} * \delta\left(a_{n}\right)\right]^{m} \quad \text { for } m=1,2, \ldots
$$

and $A_{n}$ being an automorphism of $\mathscr{P}(V)$, this last equation means

(ii) $\mu^{m}=\lim _{n} A_{n} \mu^{m n} * \delta\left(m \cdot a_{n}\right)$.

Taking the arithmetic subsequence $\{n m \mid n=1,2, \ldots\}$ of $\{n \mid n=1,2, \ldots\}$, we obtain from (i) that

(iii) $\mu=\lim _{n} A_{n m} \mu^{n m} * \delta\left(a_{n m}\right)$.

Let $\mu_{n}=A_{n m} \lambda^{n m} * \delta\left(a_{n m}\right)$. By (iii),

(iv) $\mu_{n} \rightarrow \mu$.

Equation (ii) can now be written

(v) $C_{n} \mu_{n} * \delta\left(c_{n}\right) \rightarrow \mu^{m}$

where $C_{n}=A_{n} A_{n m}^{-1}$ and $c_{n}=m \cdot a_{n}-A_{n} A_{n m}^{-1} a_{n m}$. Since $\mu$ and $\mu^{m}$ are full measures, the compactness lemma can be invoked to infer from (iv) and (v) that $\mu$ and $\mu^{m}$ are of the same type, for every positive integer $n$.

Our next aim is to extend the last theorem to include all real positive powers of $\mu$. In so doing, we get more information about the affine transformations which appear.

THEOREM 2. If $\mu$ is full and operator-stable, there is an automorphism $B$ of $V$ such that

$$
\mu^{t}=\exp \{\log t \cdot B\} \mu * \delta(b(t)) ; \quad t>0,
$$

for some $b(t) \in V$. The converse is clearly true.

While the proof is not at all difficult, it involves many steps, and for better organization, we proceed with a sequence of simple lemmas. To start with, define $G_{t}=\left\{A \in\right.$ Aut $V \mid \mu^{t}=A \mu * \delta(a)$ for some $\left.a \in V\right\}$. The set $G_{t}$ may, a priori, be empty for some $t>0$, but the last theorem shows that $G_{t}$ is nonempty whenever $t$ is a positive integer.

LeMma 1. $G_{t} \neq \varnothing$ for any $t>0$.

Proof. If $t>0$ is rational and equal to $j / k, G_{j}$ and $G_{k}$ are nonempty and $\mu^{j}$ and $\mu^{k}$ are of the same type. Therefore, there is an affine transformation $(B, b)$ such that 
$\mu^{j}=B \mu^{k} * \delta(b)$. But then, $(B \mu * \delta(1 / k \cdot b))^{k}=\mu^{j}$, and so $B \mu * \delta(1 / k \cdot b)$ is the unique infinitely divisible $k$ th root of $\mu^{j}$, so $B \mu * \delta(1 / k \cdot b)=\mu^{j / k}=\mu^{t}$. Thus, $G_{t} \neq \varnothing$ if $t$ is rational, and $\mu^{t}$ is of the same type as $\mu$ if $t$ is rational. If $t$ is any positive real number, let $t_{n}$ be rational, and let $t_{n} \rightarrow t$. Then $\mu^{t_{n}} \rightarrow \mu^{t}$ and, if

$$
\mu=C_{n} \mu^{t_{n}} * \delta\left(c_{n}\right), \quad\left(C_{n}, c_{n}\right) \in \text { Aff } V,
$$

the compactness lemma implies that $\mu^{t}$ is of the same type as $\mu$, so that $G_{t} \neq \varnothing$.

LEMMA 2. $G_{t}^{-1}=G_{1 / t}$ and $G_{s \cdot t}=G_{s} \cdot G_{t}$ for all $s, t>0$.

Proof. It is easy to see that $G_{t}^{-1} \subset G_{1 / t}$ and $G_{s} \cdot G_{t} \subset G_{s t}$. Replacing $t$ by $1 / t$ in the first inclusion and $s$ and $t$ by $1 / s$ and $s t$ in the second, the reverse inclusions hold.

LEMMA 3. $G_{s} \cap G_{t}=\varnothing$ if $s \neq t$.

Proof. If $A \in G_{s} \cap G_{t}$, say $\mu^{s}=A \mu * \delta(a)$ and $\mu^{t}=A \mu * \delta\left(a^{\prime}\right)$, then ${ }^{\circ} \mu^{s}={ }^{\circ} \mu^{t}$ implying $\left|\mu^{\wedge}(y)\right|^{2 s} \equiv\left|\mu^{\wedge}(y)\right|^{2 t}$ for all $y$. If $s \neq t$, this implies that $\left|\mu^{\wedge}(y)\right|=0$ or 1 , so $\left|\mu^{\wedge}(y)\right| \equiv 1, \mu^{\wedge}$ being continuous. But this would mean that $\mu$ is degenerate.

LEMMA 4. $G=\bigcup\left\{G_{t} \mid t>0\right\}$ is a closed subgroup of Aut $V$.

Proof. That $G$ is a subgroup is the content of Lemma 2. To prove that $G$ is closed in Aut $V$, let us assume that a sequence $\left\{A_{n}\right\}$ of members of $G$ converges in Aut $V$ to an automorphism $A$. We must show $A \in G$. Suppose $A_{n} \in G_{t_{n}}$. If $\left\{t_{n}\right\}$ contains a subsequence which converges to 0 in $R$ it may be assumed for the purposes of this argument that $t_{n} \rightarrow 0$. Then $A_{n} \mu=\mu^{t_{n}} * \delta\left(a_{n}\right)$ for some $a_{n} \in V$, so $A_{n}\left({ }^{\circ} \mu\right)=\left({ }^{\circ} \mu\right)^{t_{n}} \rightarrow \delta(0)$ as $n \rightarrow \infty$. Thus, $A\left({ }^{\circ} \mu\right)=\delta(0)$, and since ${ }^{\circ} \mu$ is full, $A=0$, a contradiction which establishes the fact that $\left\{t_{n}\right\}$ cannot have a subsequence tending to 0 . On the other hand, if $t_{n}$ has a subsequence tending to $\infty$, by setting $B_{n}=A_{n}^{-1} \in G_{1 / t_{n}}, B_{n} \rightarrow A^{-1}$ and $1 / t_{n} \rightarrow 0$. The last argument implies that $A^{-1}=0$. We conclude that $\left\{t_{n}\right\}$ must be bounded away from 0 and $\infty$. Let $t$ be any limit point of $\left\{t_{n}\right\}$; it may be assumed that $t_{n} \rightarrow t$. Then $A_{n} \mu \rightarrow A \mu$ but

$$
A_{n} \mu=\mu^{t_{n}} * \delta\left(a_{n}\right) \text { and } \mu^{t_{n}} \rightarrow \mu^{t} .
$$

Since $A \mu$ and $\mu$ are full, the compactness lemma implies $\left\{a_{n}\right\}$ is precompact in $V$, so if $a$ is any limit point, we have

$$
A \mu=\mu^{t} * \delta(a), \quad \text { so that } A \in G_{t} \subset G \text {. }
$$

Lemma 5. The mapping $\eta: G \rightarrow R^{+}$, defined by $\eta(A)=t$ if $A \in G_{t}$, is well defined, and is a continuous open homomorphism of $G$ onto the positive reals under multiplication. The kernel of $\eta$ is $G_{1}$, a compact normal subgroup of $G$.

Proof. The mapping $\eta$ is well defined by Lemma 3. It is a homomorphism because of Lemma 2. To prove that $\eta$ is continuous, suppose $A_{n} \rightarrow A_{0}$ in $G$, with 
$\eta\left(A_{n}\right)=t_{n}$, so that $A_{n} \in G_{t_{n}}$. We have $\mu^{t_{n}}=A_{n} \mu * \delta\left(a_{n}\right)$ for some $a_{n} \in V$, so $\left({ }^{\circ} \mu\right)^{t_{n}}$ $=A_{n}\left({ }^{\circ} \mu\right) \rightarrow A_{0}\left({ }^{\circ} \mu\right)-\left({ }^{\circ} \mu\right)^{t_{0}}$. But this means $\left|\mu^{\wedge}(y)\right|^{2 t_{n}} \rightarrow\left|\mu^{\wedge}(y)\right|^{2 t_{0}}$, hence $t_{n} \rightarrow t_{0}$. The openness of $\eta$ is now an automatic consequence of its continuity and the $\sigma$-compactness of $G$-see, e.g. Theorem 5.29 of Hewitt and Ross [2].

Proof of Theorem 2. The mapping $\xi=\log \eta$ is a real additive continuous homomorphism of $G$ onto $(R,+)$. Firstly, we demonstrate the existence of a oneparameter subgroup $H$ of $G$ with $\xi(H)=R$. Since $G$ is a closed subgroup of $\mathrm{Gl}(V)$, it is a linear Lie group, so the component $G^{\circ}$ of the identity in $G$ is an open normal subgroup of $G$. Since, by Lemma $5, \eta$ is open, $\xi$ is open, implying that $\xi\left(G^{\circ}\right)$ is an open subgroup of $(R,+)$ and hence must itself be $R$. Since $G^{\circ}$ is the union of its one-parameter subgroups, one of them, $H$ say, must map onto $R$ under the mapping $\xi$. Now, $H$, being a one-parameter subgroup of $\mathrm{Gl}(V)$, must be of the form $\left\{e^{s B} \mid-\infty<s<\infty\right\}$ for some operator $B$. The mapping $s \rightarrow e^{s B}$ is a continuous homomorphism of $(R,+)$ into $G$, so $s \rightarrow e^{s B} \rightarrow \xi\left(e^{s B}\right)$ is a continuous homomorphism of $(R,+)$ onto $(R,+)$ so that $\xi\left(e^{s B}\right)=K s$ for some constant $K \in R$. Replacing $B$ by $K^{-1} B$, we may assume that $\xi\left(e^{s B}\right)=s$, giving $\eta\left(e^{s B}\right)=e^{s}$ or $\eta\left(e^{\log t \cdot B}\right)$ $=t$. Thus $\exp \{\log t \cdot B\} \in G_{t}$ for every $t>0$.

It remains only to show that $B$ is invertible, if $\mu$ is full. If $B$ were noninvertible, $B^{*}$ would be noninvertible and there would exist $y \in V^{\wedge}$ such that $B^{*} y=0, y \neq 0$. In this case, $\exp \left\{\log t \cdot B^{*}\right\} y=y$ for all $t>0$, implying

$$
\left|\mu^{\wedge t}(s y)\right|=\left|\mu^{\wedge}\left(\exp \left\{\log t \cdot B^{*}\right\} s y\right)\right|=\left|\mu^{\wedge}(s y)\right| .
$$

This would mean $\left|\mu^{\wedge}\right| \equiv 1$ on the subspace generated by $y$, a contradiction to the fullness of $\mu$, by Proposition 1 .

REMARK. We shall denote $\exp \{\log t \cdot B\}$ by the notation $t^{B}$.

7. The class $\mathscr{B}$. The class of operators, $B$, which can occur in a representation $u^{t}=t^{B} \mu * \delta(b(t))$ for some full measure $\mu$ will be denoted by $\mathscr{B}$. So far, we know only that $\mathscr{B}$ consists solely of nonsingular operators. It is easy to check, now, that if $B \in \mathscr{B}$ and $A$ is any automorphism, $A B A^{-1} \in \mathscr{B}$. In other words, $\mathscr{B}$ is closed under similarity transformations, and $\mathscr{B}$ will be describable through spectral properties. In fact,

THEOREM 3. Necessary and sufficient conditions for an operator $B$ to be in the class $\mathscr{B}$ are

(i) The spectrum of $B$ is in the half-plane $\operatorname{Re} z \geqq 1 / 2$, and

(ii) The eigenvalues lying on the line $\operatorname{Re} z=1 / 2$ are simple-i.e. the elementary divisors of $B$ associated with these eigenvalues are of first degree.

Once again, the rather complicated proof obliges us to break it down to more organizable parts. Firstly, we record some facts about the representing triple of a stable distribution. 
Proposition 5. If $\lambda \in S$ has representing triple $(c, \phi, M)$ and $\lambda^{t}=t^{B} \lambda * \delta(b(t))$, $B \in \mathscr{B}$, then

(a) $\phi\left(t^{B^{*}} y\right)=t \phi(y)$ for $y \in V^{\wedge}$ and $t>0$,

(b) $t^{B} M=t M$ for $t>0$.

Proof. A direct application of Theorem 2 on Proposition 3.

The heart of the proof of Theorem 3 lies in

LEMMA 6. A measure $M$ concentrated on an orbit $\left\{t^{B} x_{0} \mid t>0\right\}$ and satisfying $t^{B} M=t M$ is $a \mathrm{~K}-\mathrm{L}$ measure if and only if every eigenvalue of $B$ in the cyclic subspace generated by $x_{0}$ has real part greater than $1 / 2$.

Proof. Assume firstly that $S(M) \subset\left\{t^{B} x_{0} \mid t>0\right\}$ and $t^{B} M=t M$. Let $X$ $=\left[x_{0}, B x_{0}, B^{2} x_{0}, \ldots\right]=\left[x_{0}\right]$, the cyclic subspace generated by $x_{0}$. Since $B$ is nonsingular, $B X=X$, and since our interest is only in the behavior of $B$ on $X$, we assume $B=B \mid X . B$ is then a cyclic operator, and by structure theory for such operators (see, e.g. Jacobson [3, p. 73]), to each elementary divisor of $B$, there is a subspace $X_{j}$, such that

(i) $B X_{j}=X_{j}$, and

(ii) $X=X_{1} \oplus \cdots \oplus X_{k}$.

Also, the minimum polynomial $q_{j}^{m}$ of $B \mid X_{j}$ is a power of a polynomial which is irreducible over the real field.

Now, set $F(s)=M\left\{t^{B} x_{0} \mid t>s\right\}$. The condition $t^{B} M \equiv t M$ implies that $F(s t)$ $=1 / t F(s)$. Thus, $F(t)=K / t$ for some constant $K>0$. The measure $M$ is a $\mathrm{K}-\mathrm{L}$ measure if and only if $\int\|x\|^{2} M(d x)<\infty$ in a neighborhood of 0 . This is the case if and only if

$$
\int_{0}^{1}\left\|t^{B} x_{0}\right\|^{2}(-d F / d t) d t<\infty
$$

or

$$
\int_{0}^{1}\left\|t^{B} x_{0}\right\|^{2} t^{-2} d t<\infty
$$

Here, $\|\cdot\|$ is any vector norm on $X$. Any other vector norm would suffice, for all such norms are equivalent on $X$. We shall specify a norm on $X$ which facilitates computation. Let $\|\cdot\|_{j}$ be a vector norm on $X_{j}$, to be chosen later, and let $\left\|\sum x_{j}\right\|$ $=\sum\left\|x_{j}\right\|_{j}$. Then $\|\cdot\|$ is a norm on $X$ with the property that $\left\|\sum x_{j}\right\| \geqq\left\|x_{r}\right\|_{r}$ for each $r, 1 \leqq r \leqq k$. Suppose $x_{0}=\sum_{j=1}^{k} x_{j}, x_{j} \in X_{j}$. Then $x_{j} \neq 0$ for each $j$, otherwise $x_{0}$ fails to be cyclic in $X$. We have then $t^{B} x_{0}=\sum_{j=1}^{k} t^{B} x_{j}=\sum_{j=1}^{k} t^{B_{j}} x_{j}$, where $B_{j}=B \mid X_{j}$, and $\left\|t^{B} x_{0}\right\| \geqq\left\|t^{B_{r}} x_{r}\right\|_{r}$, for each $r$. For the rest of the proof, let $r$ be arbitrary but fixed, $1 \leqq r \leqq k$. We now choose $\|\cdot\|_{r}$ in $X_{r}$ as follows: extend $X_{r}$ to its complexification $X_{r}^{c}$, and let $B_{r}^{c}$ be the extension of $B_{r}$ to $X_{r}^{c}$ in the usual way. We shall define a norm $\|\cdot\|_{r}^{c}$ for $X_{r}^{c}$ and let $\|\cdot\|_{r}$ be the restriction of $\|\cdot\|_{r}^{c}$ to $X_{r}$. For notational convenience, let $J\left(a_{1}, \ldots, a_{k}\right)$ denote the $k \times k$ matrix having all entries equal to zero below the diagonal, $a_{1}$ on the principal diagonal, $a_{2}$ on the super-diagonal, 
$\ldots, a_{k}$ in $(1, k)$ position. To choose $\|\cdot\|_{r}^{c}$, let us firstly choose a (complex) basis $\left\{\xi_{1}, \ldots, \xi_{p}\right\}$ for $X_{r}^{c}$ so that the matrix of $B_{1}^{c}$ with respect to $\left\{\xi_{1}, \ldots, \xi_{p}\right\}$ is $J(\alpha, 1,0, \ldots, 0)$ if $q_{j}$ is linear, and

$$
\left(\begin{array}{cc}
J(\alpha, 1,0, \ldots, 0) & 0 \\
0 & J(\bar{\alpha}, 1,0, \ldots, 0)
\end{array}\right)
$$

if $q_{j}$ is quadratic. The complex numbers $\alpha, \bar{\alpha}$ are the eigenvalues of $B_{j}$, hence of $B$. Choose $\|\cdot\|_{r}^{c}$ by making $\left\|\sum \alpha_{j} \xi_{j}\right\|_{r}^{c}=\sum\left|\alpha_{j}\right|$. Then, we have $\left\|t^{B_{r}} x_{r}\right\|_{r}=\left\|t^{B_{r}} x_{r}\right\|_{r}^{c}$, and since the (complex) matrix of $t^{B_{r}}$ can be seen by an easy computation to be

$$
J\left(t^{\alpha}, t^{\alpha} \log t, \ldots, 1 /(p-1) ! t^{\alpha}(\log t)^{p-1}\right)
$$

if $q_{j}$ is linear, or the obvious extension if $q_{j}$ is quadratic, $\left(\left\|t_{r}^{B_{r}} x_{r}\right\|_{r}^{c}\right)^{2}$ is seen to be a linear combination, with coefficients depending on $x_{r}$, of terms of the form $t^{\alpha}(\log t)^{m} t^{\bar{\alpha}}(\log t)^{n}, m \leqq p-1, n \leqq p-1$, and these terms are each $t^{2 \operatorname{Re} \alpha}(\log t)^{q}$, for some $q \leqq 2 p-2$. Then

$$
\begin{aligned}
\int_{0}^{1}\left\|t^{B_{r}} x_{r}\right\|^{2} t^{-2} d t & \geqq \int_{0}^{1} \text { const } t^{2 \operatorname{Re} \alpha} t^{-2} d t \\
& \geqq \text { const } \int_{0}^{1} t^{2 \operatorname{Re} \alpha-2} d t .
\end{aligned}
$$

The first term is finite, by (*), so $2 \operatorname{Re} \alpha-2>-1$, implying $\operatorname{Re} \alpha>1 / 2$.

To obtain the converse, note that $\operatorname{Re} \alpha>1 / 2$ implies $\int_{0}^{1} t^{2 \operatorname{Re} \alpha-2} d t<\infty$ and an integration by parts shows that all the terms $t^{\alpha}(\log t)^{m} t^{\bar{\alpha}}(\log t)^{n}$, as above, have finite integrals at 0 . Taking the same norms as above, we find

so

$$
\int_{0}^{1}\left\|t^{B_{r}} x_{r}\right\|_{r}^{2} t^{-2} d t<\infty
$$

$$
\int_{0}^{1}\left\|t^{B} x\right\|^{2} t^{-2} d t \leqq \text { const } \Sigma_{r} \int_{0}^{1}\left\|t^{B_{r}} x_{r}\right\|^{2} t^{-2} d t<\infty .
$$

We now proceed with the

Proof of Theorem 3. Let $\Lambda(B)=\left\{M \mid M\right.$ a K-L measure on $V \sim\{0\}$ and $\left.t^{B} M=t M\right\}$. Note that if $M \in \Lambda(B), S(M)$ is invariant under $t^{B}$ for all $t>0$, so that $S(M)$ is a union of orbits of $t^{B}$. The $M$ may be infinite measures, but they are essentially finite in the sense that $W(d x)=\|x\|^{2} /\left(1+\|x\|^{2}\right) M(d x)$ is a finite measure in $V$. To apply the theory of finite measures, let $\mathfrak{M}(V)$ be the real linear space of finite measures in $V$, with the topology of weak convergence. Let

$$
\Omega(B)=\left\{W \in \mathfrak{M}(V) \mid W(d x)=\|x\|^{2} /\left(1+\| x_{\|}^{2}\right) M(d x), M \in \Lambda(B)\right\} .
$$

$\Omega(B)$ is easily seen to be a convex cone in $\mathfrak{M}$, and $\Omega_{1}(B)=\{W \in \Omega \mid W(V) \leqq 1\}$ is a compact convex subset of $\mathfrak{M}$. Each $W$ in $\Omega$ has the property that $S(W)$ is a union of orbits of $t^{B}$. Thus, it is easily seen that the extreme points of $\Omega_{1}(B)$ are the 
measures concentrated along a single orbit $\left\{t^{B} x_{0} \mid t>0\right\}$. Thus, the set of convex combinations of such measures is dense in $\Omega_{1}$, and this shows that in $\Lambda(B)$, the linear combinations of $M$ 's which are concentrated in a single orbit are dense in $\Lambda(B)$.

Let $X$ now be decomposed into a direct sum of subspaces $X_{j}(1 \leqq j \leqq r)$ such that $B X_{j}=X_{j}$, and such that the minimum polynomial of $B \mid X_{j}$ is a power of a realirreducible polynomial. Assume that the eigenvalues in $X_{1}, \ldots, X_{k}$ lie in $\operatorname{Re} z>1 / 2$ and those in $X_{k+1}, \ldots, X_{r}$ lie in $\operatorname{Re} z \leqq 1 / 2$. Let $X_{0}=X_{k+1}+\cdots+X_{r}$. Now, if $x \in X$, and $x=\sum_{0}^{k} x_{j}, x_{j} \in X_{j}$ then, by Lemma 6 , the orbit $\left\{t^{B} x \mid t>0\right\}$ supports a nonzero K-L measure $M \in \Lambda(B)$ if and only if $x_{0}=0$. Hence, since linear combinations of such $M$ are dense in $\Lambda(B), M$ is concentrated in $X_{1}+\cdots+X_{k}$, for all $M \in \Lambda(B)$.

To find how $B$ behaves in $X_{0}$, examine the adjoint $B^{*}: V^{\wedge} \rightarrow V^{\wedge}$. Let, in $V^{\wedge}$,

$$
Y_{j}=\left(X_{0} \oplus \cdots \oplus X_{j-1} \oplus X_{j+1} \oplus \cdots \oplus X_{k}\right)^{\perp}, \quad 0 \leqq j \leqq k .
$$

$Y_{j}$ is then the dual of $X_{j}$, and $B^{*} \mid Y_{j}=\left(B \mid X_{j}\right)^{*}$. It is a consequence of the Khintchine-Lévy formula that

$$
\log { }^{\circ} \wedge(y)=-2 \phi(y)+\int(\cos (x, y)-1) M(d x)
$$

and if $y \in Y_{0}$, since $M$ is concentrated in $X_{1} \oplus \cdots \oplus X_{k}$,

$$
\log { }^{\circ} \wedge(y)=-2 \phi(y) \text {. }
$$

Since $\mu$ is assumed full, $\phi(y) \neq 0$ if $y \in Y_{0}, y \neq 0$. Thus, the quadratic form $\phi \mid Y_{0}$ is nondegenerate, and there is a basis $\left\{y_{1}, \ldots, y_{q}\right\}$ in $Y_{0}$ such that

$$
\phi\left(\sum_{1}^{q} a_{j} y_{j}\right)=\sum_{1}^{q} a_{j}^{2}
$$

With respect to this basis $\left\{y_{1}, \ldots, y_{q}\right\}$, a bilinear form $\langle\cdot, \cdot\rangle$ is defined, and adjoints $C^{\prime}$ of operators $C$ are defined by

$$
\left\langle C y_{1}, y_{2}\right\rangle=\left\langle y_{1}, C^{\prime} y_{2}\right\rangle \text {. }
$$

Then, the condition 5(a) reads that for all $y \in Y_{0}$ and $t>0$,

$$
\phi\left(t^{B^{*}} y\right)=t \phi(y) \text {. }
$$

Since $B^{*} Y_{0}=Y_{0}$, it will not hurt to assume in this section that $B^{*}=B^{*} \mid Y_{0}$. Then, for all $y \in Y_{0}$,

Thus,

$$
\left\langle t^{B^{*}} y, t^{B^{*}} y\right\rangle=t\langle y, y\rangle \text {. }
$$

or

$$
\left\langle t^{B^{*}} t^{B^{\prime}} y, y\right\rangle=\langle t y, y\rangle
$$

$$
t^{B^{*}} t^{B^{\prime}} \equiv t I \equiv t^{1 / 2} I \cdot t^{1 / 2} I \text {. }
$$

Since $t^{1 / 2} I \equiv t^{I / 2}$, this can be written as

$$
t^{\left(B^{*}-I / 2\right)} t^{\left(B^{*}-I / 2\right)^{\prime}} \equiv I .
$$


Setting $C=B^{*}-I / 2$, we find that $t^{C+C^{\prime}} \equiv I$ for all $t>0$, so that $C+C^{\prime}=0$. Thus, in this basis, $C$ is a skew operator. Hence $B^{*}=I / 2+C$ is a normal operator, and all its elementary divisors are linear. This proves the necessity of the conditions in the theorem. To prove the sufficiency of the conditions, let $B$ satisfy (i) and (ii). As in the proof of the necessity, let $X=X_{1} \oplus \cdots \oplus X_{r}, X_{j}$ having the same meaning as before. If $x_{j} \in X_{j}, x_{j} \neq 0,(1 \leqq j \leqq k)$, then, by Lemma 6 , the orbit $\left\{t^{B} x_{j} \mid t>0\right\}$ supports a nonzero K-L measure $M_{j} \in \Lambda(B)$. Since the orbit $\left\{t^{B} x_{j} \mid t>0\right\}$ generates $X_{j}$, the stable measure $\lambda_{j}$ with triple $\left(0,0, M_{j}\right)$ is supported, and is full, in $X_{j}$. On $X_{0}=X_{k+1} \oplus \cdots \oplus X_{r}$, we construct a Gaussian measure $\mu$ satisfying $\mu^{t}$ $=\exp \left\{\log t \cdot B \mid X_{0}\right\} \mu$. We can imagine that $X=X_{0}$, hence that $B$ may be put into a canonical form $\operatorname{diag}\left\{J_{1}, \ldots, J_{q}\right\}$ where $J_{j}$ is either a $1 \times 1$ matrix with element $1 / 2$, or a $2 \times 2$ matrix

$$
\left(\begin{array}{cr}
1 / 2 & \beta \\
-\beta & 1 / 2
\end{array}\right) .
$$

With the dual basis $\left\{\eta_{1}, \ldots, \eta_{p}\right\}$ in $Y_{0}, B^{*}$ has canonical form $\operatorname{diag}\left\{J_{1}^{*}, \ldots, J_{q}^{*}\right\}$. We have to construct a quadratic form $\phi$ on $Y_{0}$ such that $\phi\left(t^{B^{*}} y\right) \equiv t \phi(y)$. Consider $\phi\left(\sum a_{i} \eta_{i}\right)=\sum a_{i}^{2}$. If $\left[\eta_{1}\right]=X_{j}, t^{J_{j}}=t^{1 / 2} I$ and $\phi\left(t^{J_{j}} a \eta_{i}\right)=t \phi\left(a \eta_{i}\right)$. If $\left[\eta_{i}, \eta_{i+1}\right]=X_{j}$, we have

$$
t^{J_{j}}=t^{1 / 2}\left(\begin{array}{rr}
\cos (\beta \log t) & \sin (\beta \log t) \\
-\sin (\beta \log t) & \cos (\beta \log t)
\end{array}\right)
$$

and, once again, $\phi\left(t^{J},\left(a \eta_{i}+b \eta_{i+1}\right)\right)=t \phi\left(a \eta_{i}+b \eta_{i+1}\right)$. Thus, $e^{-\phi(y)}$ is a Gaussian characteristic function, and its distribution is full and concentrated in $X_{0}$. The measure $\nu=\lambda_{1} * \cdots * \lambda_{k} * \mu$ is now full and stable, and $\nu^{t}=t^{B} \nu$.

As by-products of the proof of Theorem 3, we can assert the following:

THEOREM 4. Any full operator-stable measure $\lambda$ on $V$ can be decomposed into a product $\lambda=\lambda_{1} * \lambda_{2}$ of measures $\lambda_{i}$ concentrated in subspaces $V_{i}, V=V_{1} \oplus V_{2}$, where $\lambda_{1}$ is a full Gaussian measure in $V_{1}$ and $\lambda_{2}$ is a full operator-stable measure on $V_{2}$ having no Gaussian component.

THEOREM 5. Any K-L measure $M$ for a full operator-stable measure $\lambda$ on $V$ can be represented as a mixture of $\mathrm{K}-\mathrm{L}$ measures $M_{\theta}$ where $M_{\theta}$ is a K-L measure concentrated in an orbit, $\theta$, of $t^{B}$ and satisfies $t^{B} M_{\theta}=t M_{\theta}$. The measure $M_{\theta}$ is characterized by the condition that $s M_{\theta}\left\{t^{B} x_{0} \mid t>s\right\}$ is constant for all $s$, when $x_{0}$ is a generator of the orbit $\theta$.

If $\lambda$ is full and operator-stable, and satisfies $\lambda^{t}=t^{B} \lambda * \delta(b(t))$ for some $b(t)$, we shall call $B$ an exponent for $\lambda$. A measure $\lambda$ may possess more than one exponent. For example, if $V$ is given an inner product with respect to which $\lambda$ is a rotationinvariant Gaussian measure, then $B=I / 2$ is an exponent for $\lambda$, as is any operator of the form $I / 2+C$ where $C$ is skew.

The operators in the class $\mathscr{B}$ can be interpreted as exponents of the normalizing factors which give rise to operator-stable laws. That is, if $x_{1}, x_{2}, \ldots$ are independent 
full operator-stable random variables having the same distribution, then each has the same distribution as some translate of $n^{-B}\left(x_{1}+\cdots+x_{n}\right)$, for $n=1,2, \ldots$

8. Centering. We turn, finally, to an examination of the term $b(t)$ in the formula

$$
\lambda^{t}=t^{B} \lambda * \delta(b(t)) \text { for all } t>0,
$$

satisfied by a full operator-stable measure $\lambda$ with exponent $B$. Taking sth powers on both sides of (8.1), we find that

$$
\lambda^{s t}=t^{B} \lambda^{s} * \delta(s b(t))=t^{B} s^{B} \lambda * \delta\left(t^{B} b(s)+s b(t)\right) .
$$

But $\lambda^{s t}=(s t)^{B} \lambda * \delta(b(s t))$, from (8.1), so that the vector-valued function $b(\cdot)$ must satisfy the functional equation

$$
b(s t)=t^{B} b(s)+s b(t) \text { for all } s>0, t>0 .
$$

One consequence, obtained by setting $s=t=1$, is that $b(1)=0$.

THEOREM 6. If 1 is not in the spectrum of $B$, the general solution of $(8.2)$ is

$$
b(t)=t x_{0}-t^{B} x_{0}, \quad t>0,
$$

for some $x_{0} \in V$, and in this case, when the full operator-stable measure $\lambda$ satisfying (8.1) is centered at $x_{0}$, the measure $\mu=\lambda * \delta\left(-x_{0}\right)$ satisfies $\mu^{t}=t^{B} \mu$.

REMARK. By analogy with the one-dimensional case, an operator-stable measure $\mu$ satisfying $\mu^{t}=t^{B} \mu$ could be called strictly stable. Our theorem shows that when 1 is not in the spectrum of $B$, any full operator-stable measure can be centered so as to become strictly stable.

Proof. Once we have proven (8.3), the second assertion of the theorem follows by a standard calculation. As a first step in proving (8.3), note that if $b_{1}(\cdot)$ and $b_{2}(\cdot)$ are solutions of $(8.2)$, then $b_{1}(\cdot)-b_{2}(\cdot)$ is a solution of $(8.2)$. Note also that if $b(\cdot)$ is a solution of (8.2) such that $b\left(t_{0}\right)=0$ for some $t_{0} \neq 1$, then

$$
b\left(s t_{0}\right)=t_{0} b(s)=t_{0}^{B} b(s) \text { for all } s>0 .
$$

Since 1 is not an eigenvalue of $B, t_{0}$ is not an eigenvalue of $t_{0}^{B}$, so we must have $b(s) \equiv 0$. These observations imply that any two solutions of (8.2) which agree at even one point must in fact be identical.

Now, the operator $\left(t I-t^{B}\right)$ is invertible, for $t \neq 1$, hence we can always solve an equation

$$
\left(t I-t^{B}\right) x_{0}=x_{1} \quad \text { for } x_{0} \in V \text {, for all } x_{1} \in V .
$$

Hence, the solutions $b(t)=t x_{0}-t^{B} x_{0}$ constitute all possible solutions of (8.2).

In the event that $B$ has 1 as an eigenvalue, it may not be possible to center $\lambda$ as in the last theorem. This is in analogy with the one-dimensional case of the so-called asymmetric Cauchy distribution. An example is given by taking $B=I$ so that (8.2) becomes

$$
b(s t)=s b(t)+t b(s),
$$


which is satisfied by the function $b(t)=t \log t x_{0}$, for all $x_{0} \in V$. We can clearly not absorb such a factor to center $\lambda$.

ACKNOWLEDGEMENT. I wish to express my sincere gratitude to my advisor, Shizuo Kakutani, for the help he has given me during the preparation of this paper.

\section{REFERENCES}

1. W. Feller, An introduction to probability theory and its applications, Vol. II, Wiley, New York, 1966.

2. E. Hewitt and K. Ross, Abstract harmonic analysis, Vol. I, Springer-Verlag, Berlin, 1963.

3. N. Jacobson, Lectures in abstract algebra, Vol. II, Van Nostrand, Princeton, N. J., 1953.

4. P. Lévy, Théorie de l'addition des variable aléatoires, 2nd ed., Gauthier-Villars, Paris, 1954.

University of California at San Diego,

la Jolla, California

YALE UNIVERSITY,

New Haven, Connecticut 